\title{
Measurement of functional independence level and falls-risk in individuals with undiagnosed phenylketonuria
}

\author{
Artur Mazur ${ }^{\circledR}$, Sabina Jarochowicz ${ }^{1}$, Mariusz Ołtarzewski², Jolanta Sykut-Cegielska ${ }^{3}$, \\ Andrzej Kwolek ${ }^{1}$ and Grace $\mathrm{O}^{\prime} \mathrm{Malley}^{4}$ \\ ${ }^{1}$ Medical Faculty, University of Rzeszów, Poland, ${ }^{2}$ Institute of Mother and Child, Warszawa, Poland, \\ ${ }^{3}$ Children's Memorial Health Institute in Warszawa, Poland, ${ }^{4}$ Department of Physiotherapy, \\ The Children's University Hospital, Dublin, Ireland
}

Received: 30 January, 2009; revised: 07 October, 2009; accepted: 19 October, 2009

available on-line: 08 December, 2009

\begin{abstract}
The aim of the study was to determine the level of functional independence in adult patients with previously undiagnosed or untreated phenylketonuria (PKU). The study was conducted among 400 intellectually impaired adult residents of Social Welfare Homes in South-Eastern Poland born prior to the introduction of neonatal PKU screening programs. PKU was screened by filter paper test using tandem mass spectrometry methods, and confirmed by gas chromatography-mass spectrometric analysis of PKU organic acids in urine. Degree of functional independence included the assessment of activities of daily living (Barthel Index) and measures of balance and gait (Tinetti scale). Eleven individuals with previously untreated PKU were identified whereby eight presented with moderate disability and three with mild disability. Six had a high risk of falls and five had a moderate risk of falls. This study indicates that there is considerable number of undiagnosed PKU patients within the Polish population who require assessment and management in order to reduce the impact of the neurological and neuropsychiatric problems associated with the condition. Appropriate therapy for those with undiagnosed PKU should, in particular, address the risk of falls.
\end{abstract}

Keywords: undiagnosed PKU, falls, functional independence level, balance, gait

\section{INTRODUCTION}

Over the last 70 years the clinical picture of phenylketonuria (PKU) has been evolving, with patients who were untreated in the past and presenting with severe intellectual and physical disabilities as starting point. Thanks to the implementation of PKU screening, there are today thousands of individuals who, due to a timely diagnosis and subsequent treatment, can fulfill their intellectual and physical potentials. Although newborn screening has been in use for over 40 years in Poland, there exists a group of adults whose multiple health problems related to remote consequences of neurotoxic phenylalanine (Phe) effects occur, due to a lack of or a delay in medical intervention (Rolling, 1984; Zekanowski et al., 2000; Kasim et al., 2001; Giżewska, 2006). To date, very few coordinated actions have been undertaken in Poland in order to detect this particular group of patients (Giżewska, 2006). The primary aim of the present study was to evaluate the level of functional independence of patients with previously undiagnosed or untreated PKU. A secondary aim was to determine whether these individuals demonstrated balance and gait impairments which could possibly increase their risk of falling.

\section{MATERIALS AND METHODS}

Study population. The study was carried out from December 2006 to April 2007 in 12 Social Welfare Homes for intellectually disabled people in South-Eastern Poland. Patients were selected for

${ }^{\square}$ Corresponding author: Artur Mazur, Institute of Physiotherapy, University of Rzeszów, Warszawska 26a, 35-205 Rzeszów, Poland; tel.: (48) 17866 4244; fax: (48) 17866 4145; e-mail: drmazur@poczta.onet.pl Abbreviations: ADL, activities of daily living; PKU, phenylketonuria. 
screening if they were born prior to obligatory neonatal PKU screening (1976) and if they exhibited symptoms of classical PKU.

Such symptoms include: fair colouring of the hair, skin and retina; enamel hypoplasia and challenging behavior such as agitation, aggression and self-injury. In addition, residents who were suspected to be 'missed cases' or who had been previously diagnosed but remained untreated were also included in our analysis. The study was conducted in accordance with The Declaration of Helsinki and approved by the Ethical Committee of The Medical Faculty, University of Rzeszów.

Following the inclusion criteria, 398 residents were identified for screening (200 women, 198 men; mean age 51 years, age range 31-98 years). Additionally, one patient born in 1979 who exhibited symptoms of classical PKU was suspected to be a 'missed case' and therefore included into our screening procedures for PKU. Finally, during medical chart review we identified and also included into the study, one individual born in 1984 who was previously diagnosed with PKU but who remained untreated.

Screening procedure. Quantative analysis testing for Phe and Tyr using tandem mass spectrometry was performed in dried blood samples of 400 residents as described previously (Sansom, 1999). In order to confirm the diagnoses, positive test results underwent a urine profile analysis for organic acids, determined by chromatography-mass spectrometry (Chalmers \& Lawson, 1975).

Functional independence. Functional independence in activities of daily living (ADL) was assessed in 11 patients identified as having untreated PKU, using the Barthel Index (Mahoney \& Barthel, 1965). The Barthel Index is a valid measure of disability and one of the most widely used tools for quantifying functional impairment in adults (Collin et al., 1988; Hsueh et al., 2002). It determines basic independent functional ability across ten domains: feeding; grooming; dressing; bathing; toilet use; ambulation; transfers; stair climbing; bowel control and bladder control. The level of independence is determined according to whether the patient can perform these tasks independently, with assistance or with total dependence from care providers (Dalek \& Czernicki, 1999; Hsueh et al., 2002). Standardised guidelines for implementing and scoring the Barthel Index were followed as previously recommended (Mahoney \& Barthel, 1965).

Balance and gait assessment. Balance and gait in patients with untreated PKU was assessed using the Tinetti scale (Tinetti, 1986; Dalek \& Czernicki, 1999). This user-friendly tool consists of a comprehensive assessment of nervous and osteoarticular system and yields a score which can be used to estimate the risk of falls (Dalek \& Czernicki, 1999;
Tinetti, 1986). Two fundamental parts of the test analyse balance and gait abilities. In the present study, we evaluated the level of independence and motor skills among untreated PKU patients using 14 of the 16 motor tasks assessed in Tinetii Test. The first part of the testing evaluated balance disturbances and consisted of the following tasks: sitting balance, chair rising, attempts to chair rise, immediate standing balance (first five seconds), standing balance, turning 360 degrees, and sitting down. The second part related to gait and evaluated the following components: initiation of gait, step length and height, step symmetry, step continuity, path deviation of the gait, associated trunk movements, and heel position during walking.

Following the recommended guidelines, direct observation was carried out using a stool with a firm seat and without a back, a stopwatch, and a space providing walking path of at least 3 meters. Scoring of the test was completed on a three-point ordinal scale with a range from zero to two, whereby zero represented the greatest impairment, and two was awarded where the subject performed the task independently. The maximum achievable score was a total of 25 points (13 for balance and 12 for gait). By analyzing the overall gait and balance scores the falls risk was classified as high, medium or low, as recommended previously (Collin et al., 1988).

\section{RESULTS}

Among 400 residents of Social Welfare Homes included in the study, 11 patients ( 7 men and 4 women) had a positive screening test result for PKU and never been treated. Since one patient was diagnosed before, the incidence of newly detected PKU cases in the studied group was $2.5 \%$. All subjects in the cohort were White Caucasian.

Table 1. Characteristics of $\mathbf{1 1}$ detected subjects with previously untreated PKU

\begin{tabular}{|c|c|c|c|}
\hline Subject & Age (years) & Sex/gender & $\begin{array}{l}\text { Blood Phe level } \\
\text { (mg/dl) }\end{array}$ \\
\hline 1. & 59 & $\pi$ & 29.8 \\
\hline 2. & 52 & $\pi$ & 28.66 \\
\hline 3. & 46 & $\widehat{o}$ & 25.04 \\
\hline 4. & 23 & q & 24.5 \\
\hline 5. & 45 & q & 24.04 \\
\hline 6. & 50 & q & 22.38 \\
\hline 7. & 56 & q & 21.85 \\
\hline 8. & 59 & $\hat{0}$ & 21.63 \\
\hline 9. & 28 & $\hat{0}$ & 18.46 \\
\hline 10. & 55 & $\hat{\sigma}$ & 10.08 \\
\hline \multirow[t]{2}{*}{11.} & 49 & $0^{3}$ & 9.2 \\
\hline & $\begin{array}{l}\text { mean (S.D.) } \\
47.5 \text { (11.9) }\end{array}$ & & $\begin{array}{l}\text { mean (S.D.) } \\
21.4(6.6)\end{array}$ \\
\hline
\end{tabular}


Table 2. The results of independence assessment in daily life activities recorded among 11 previously untreated PKU individuals

\begin{tabular}{llcc}
\hline No. & $\begin{array}{l}\text { Activities } \\
\text { (Barthel Index) }\end{array}$ & $\begin{array}{l}\text { Mean score by group/ } \\
\text { max. Barthel Index } \\
\text { score (points) }\end{array}$ & $\begin{array}{l}\text { Range score within } \\
\text { studied group (points) }\end{array}$ \\
\hline 1. & feeding & $4.09 / 10$ & $0-5$ \\
2. & transfers & $10.45 / 15$ & $0-15$ \\
3. & grooming & $1.36 / 5$ & $0-5$ \\
4. & toilet use & $6.36 / 10$ & $0-10$ \\
5. & bathing & $0.45 / 5$ & $0-5$ \\
6. & ambulation & $12.27 / 15$ & $10-15$ \\
7. & stair climbing & $6.28 / 10$ & $5-10$ \\
8. & dressing & $4.55 / 10$ & $0-10$ \\
9. & bowel control & $7.27 / 10$ & $0-10$ \\
10 & bladder control & $7.27 / 10$ & $0-10$ \\
\hline & total index score & $60.35 / 100$ & $30-85$ \\
\hline
\end{tabular}

The oldest individual with PKU was 59 years old; while the youngest was 23 years old (mean age 47.5 years). The plasma Phe levels of the identified patients with PKU ranged between $9.52 \mathrm{mg} / \mathrm{dl}$ and $29.80 \mathrm{mg} / \mathrm{dl}$, with mean (S.D.) Phe $21.4 \mathrm{mg} / \mathrm{dl}$ (6.6), 95\% CI $(16.60,26.20)$. Table 1 describes characteristics of the cohort. In the other 399 studied subjects, the result of the screening test was negative with a mean (S.D.) Phe of $1.68 \mathrm{mg} / \mathrm{dl}(0.63)$ and $95 \%$ CI $(1.31,2.05)$. Screening identified seven cases of classic PKU (mean Phe: $24.77 \mathrm{mg} / \mathrm{dl}$ ), two cases of mild PKU (mean Phe: 14.27) and a single case of mild hyperphenylalaninemia (Phe: $9.20 \mathrm{mg} / \mathrm{dl}$ ). Previously diagnosed but untreated for PKU identified 23 year old female represented classical form of PKU disease (Phe: $24.5 \mathrm{mg} / \mathrm{dl}$ ).

The results of functional independence testing in the 11 untreated patients with PKU are presented in Table 2. Figure 1 illustrates the level of dependence on caregivers required during ADLs using the Barthel Index.

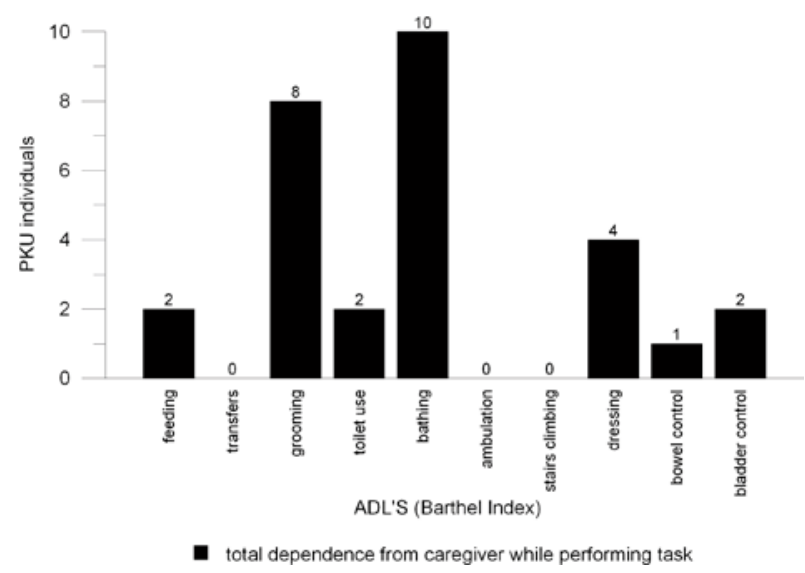

Figure 1. The characteristic of dependence in activities of daily living according to Barthel Index within studied PKU individuals.
Evaluation of each ADL task revealed that the greatest degree of dependence on caregivers was required for bathing (10 patients); grooming (combing, shaving, dental hygiene) (eight patients) and dressing/ undressing (four patients). Four of the subjects were completely independent in walking along a flat surface, whereas the other six subjects required verbal encouragement or physical assistance during the task. None of the study participants were wheelchair users. The majority of participants required assistance for stair climbing (seven patients) and only four participants completed the task independently. Three patients had continence difficulties.

The evaluation of functional mobility during ADLs indicated that none of the participants were considered to have a severe level of disability. The mean Tinetti score for the group was 14.55 points (range 9-21 points) with the mean balance and gait scores of 7.45 and 7.09 points, respectively (balance range 4-11, gait range 4-11 points). From the Tinetti scores achieved the risk of falling was estimated for each patient (Fig. 2). Detailed results of Tinetti testing are presented in Table 3.

During balance testing the most considerable activity limitations were noted during turning 360 degrees, sitting, and maintaining standing balance. Those who managed to turn 360 degrees did so in an unsteady manner and required verbal cues. The most common difficulty encountered when attempting to sit into a chair was a misjudgment of distance and a consequent uncontrolled leaning into the chair. Those who achieved standing balance displayed a broad stance and required upper limb assistance during the initial stage of standing. During gait assessment, the most common difficulties observed included hesitancy and indecision during gait initiation, deviating from the chosen path, and the co-existence of trunk movements.

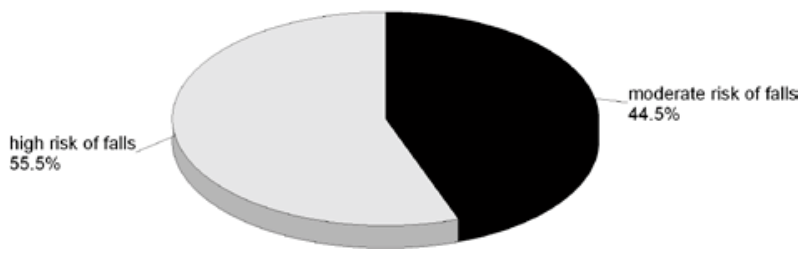

Figure 2. Results of assessment for risk of falls among 11 studied individuals with untreated PKU. 
Table 3. The results of balance and gait assessment recorded among studied PKU patients using Tinetti test

\begin{tabular}{|c|c|c|c|c|}
\hline $\begin{array}{l}\text { Mobility results } \\
\text { Motor tasks }\end{array}$ & $\begin{array}{l}\text { Scale } \\
\text { scoring }\end{array}$ & $\begin{array}{l}\text { Number of } \\
\text { PKU subjects }\end{array}$ & $\begin{array}{l}\text { Percentage of } \\
\text { PKU subjects }\end{array}$ & $\begin{array}{l}\text { Mean scoring for PKU group/max } \\
\text { possible score result (points) }\end{array}$ \\
\hline \multicolumn{5}{|l|}{ BALANCE } \\
\hline \multirow[t]{2}{*}{ Sitting balance } & 0 point & 1 & $90.9 \%$ & $0.90 / 1$ \\
\hline & 1point & 10 & $9.1 \%$ & \\
\hline \multirow[t]{3}{*}{ Arises } & 0 point & - & - & $1.63 / 2$ \\
\hline & 1 point & 4 & $36 \%$ & \\
\hline & 2 point & 7 & $64 \%$ & \\
\hline \multirow[t]{3}{*}{ Attempts to arise } & 0 point & - & - & $1.72 / 2$ \\
\hline & 1 point & 3 & $27 \%$ & \\
\hline & 2 points & 8 & $73 \%$ & \\
\hline \multirow[t]{3}{*}{ Immediate standing balance (5 s) } & 0 point & - & - & $1.55 / 2$ \\
\hline & 1point & 5 & $45 \%$ & \\
\hline & 2 points & 6 & $55 \%$ & \\
\hline \multirow[t]{3}{*}{ Standing balance } & 0 point & 3 & $27 \%$ & $\underline{0.90 / 2^{*}}$ \\
\hline & 1point & 6 & $55 \%$ & \\
\hline & 2 points & 2 & $18 \%$ & \\
\hline \multirow[t]{2}{*}{ Turning 360 degrees } & 0 point & 9 & $82 \%$ & $\underline{0.36 / 2}$ \\
\hline & 1point & 2 & $18 \%$ & \\
\hline \multirow[t]{3}{*}{ Sitting down } & 0 point & 5 & $45 \%$ & $\underline{0.54 / 2}$ \\
\hline & 1point & 6 & $55 \%$ & \\
\hline & 2 points & - & - & \\
\hline \multicolumn{5}{|l|}{ GAIT } \\
\hline \multirow[t]{2}{*}{ Initiation of gait } & 0 point & 7 & $64 \%$ & $\underline{0.36 / 1}$ \\
\hline & 1point & 4 & $36 \%$ & \\
\hline \multirow{4}{*}{$\begin{array}{l}\text { Step length and height } \\
\text { a) right swing foot }\end{array}$} & 0 point & 2 & $18 \%$ & $1.45 / 2$ \\
\hline & 1point & 9 & $82 \%$ & \\
\hline & 0 point & 4 & $36 \%$ & \\
\hline & 1point & 7 & $64 \%$ & \\
\hline \multirow{4}{*}{$\begin{array}{l}\text { Step length and height } \\
\text { b) left swing foot }\end{array}$} & 0 point & 1 & $9.1 \%$ & $1.63 / 2$ \\
\hline & 1point & 10 & $90.9 \%$ & \\
\hline & 0 point & 3 & $27 \%$ & \\
\hline & 1point & 8 & $73 \%$ & \\
\hline \multirow[t]{2}{*}{ Step symmetry } & 0 point & 3 & $27 \%$ & $0.72 / 1$ \\
\hline & 1point & 8 & $73 \%$ & \\
\hline \multirow[t]{2}{*}{ Step continuity } & 0point & 8 & $73 \%$ & $\underline{0.27 / 1}$ \\
\hline & 1 point & 3 & $27 \%$ & \\
\hline \multirow{3}{*}{$\begin{array}{l}\text { Path (estimate } 12 \text { inch floor tiles } \\
\text { over } 10 \text { feet) }\end{array}$} & 0 point & 2 & $18 \%$ & $\underline{1.18 / 2}$ \\
\hline & 1points & 5 & $45 \%$ & \\
\hline & 2 points & 4 & $36 \%$ & \\
\hline \multirow[t]{3}{*}{ Trunk (e.g. sways. spread arms) } & 0 point & 1 & $9 \%$ & $\underline{1.27 / 2}$ \\
\hline & 1 point & 6 & $55 \%$ & \\
\hline & 2 points & 4 & $36 \%$ & \\
\hline \multirow[t]{2}{*}{ Walking time (heels) } & 0 point & 9 & $82 \%$ & $\underline{0.18 / 1}$ \\
\hline & 1 point & 2 & $18 \%$ & \\
\hline
\end{tabular}

*Motor tasks which were the most troublesome for patients to perform and during which they presented the less mobility independency had been marked and underlined

\section{DISCUSSION}

We aimed to evaluate the level of functional independence of patients with previously undiagnosed or untreated PKU and to determine whether such individuals demonstrated balance and gait impairments which might increase the risk of falling. We detected 11 untreated cases of PKU in a population of 400 intel- 
lectually disabled individuals, 10 of which were newly diagnosed. As such, the prevalence of new PKU diagnoses was 2.5 cases per 100. This result supports a previous finding by Giżewska (2006), who reported the incidence of previously undiagnosed, late-diagnosed or temporarily treated PKU to be $1.7 \%$ (17 of 975 cases, mean age 40.4 years). Similarly, Brown and Guest (1999) estimated that there might be up to 2000 cases of untreated PKU in the population of Great Britain.

We evaluated the level of functional independence in the performance of ADLs in patients with untreated PKU using the Barthel Index. We determined that $73 \%$ of patients had a moderate disability and $27 \%$ had a mild disability. We are unaware of any other study in the literature that utilised standardised and valid measures to assess independence during ADLs in PKU patients.

Evaluating the level of independence is important in the managment of these patients as therapeutic interventions have shown that the annual cost of caring for patients with PKU can be reduced by decreasing the time and intensity of the nursing care required. (Brown \& Guest, 1999). Patients with untreated PKU usually develop further progressive and pathological neurological dysfunction with age, which can manifest as a deterioration in general motor functioning (Ishimaru et al., 1993; Williams, 1998; Fitzgerald et al., 2000; Kasim et al., 2001; Giżewska et al., 2003; Merrick et al., 2003). Such deteriorations include: increased muscle tone, muscle spasm, myoclonus, ataxia and paresis, all of which can lead to increased disability.

Our study is the first one to assess the balance and gait impairments manifested in patients with PKU. Using the Tinetti scale we described these impairments and the data revealed that $55.5 \%$ of the group had a high falls-risk and $45.5 \%$ a moderate falls-risk. As such, all patients were at risk of falling. Given that the estimated incidence of untreated PKU in the Polish population may lie between 1.7\% and 2.5\% (654512-962517 individuals) and considering the significant economic burden associated with falls, it would be fiscally prudent to limit, where possible, the risk of falls in individuals with untreated PKU. Considering previous reports on the benefits of treatment, such strategies are indicated in our study group, in order to improve gross neurological status and motor function (Yannicelli \& Ryan, 1995; Fitzgerald et al., 2000; Kasim et al., 2001).

Appropriate therapeutic management has been shown to be beneficial to both patients and their carers. In a prospective double-blind randomised placebo-controlled crossover trial by Lee and coworkers (Lee et al., 2009), 16 participants from a group of 34 individuals with late-diagnosed PKU and reported with severe challenging behavior completed 60 weeks of a Phe-restricted diet. Significant improvements in quality of life by patients and carers were achieved during the treatment phase compared to the placebo phase $(P<0.01)$ In addition, improvements in selfcare, continence and a reduced dependency on carers in adults with untreated PKU following the introduction of a low-Phe diet have been reported (Yannicelli \& Ryan, 1995; Giżewska, 2006). A decrease in Phe blood levels and reduced limitation in ADLs has also been reported in $34 \%$ of subjects who underwent dietary intervention (Brown \& Guest, 1999).

It is clear from the literature that the introduction of appropriate dietary changes can be beneficial to the neurological function of the previously untreated individual with PKU. As far as the authors are aware, our study is the first to present objectively measured data relating to the functional activity level of such patients. Due to the high risk of falls and physical impairments exhibited by this group, it is recommended that such patients are not only provided with dietary intervention, but that they also receive an appropriate level of physical therapy to address impairments, to maximise physical function, and to reduce the risk of falls.

We recommend that in individuals with previously untreated PKU, a client-specific treatment plan should be drawn up in agreement with the patient and his/her carers, where possible. Based on the impairments exhibited by the patient, appropriate dietary, physical, occupational and behavioural therapies should be implemented in order to improve the level of independence, enhance quality of life and reduce the healthcare costs associated with high falls-risk and nursing requirements.

\section{CONCLUSION}

This study indicates that there are a considerable number of undiagnosed PKU patients within the Polish population who require assessment and management in order to reduce the impact of the neurological and neuropsychiatric problems associated with the condition. Appropriate therapy for those with undiagnosed PKU should in particular address the risk of falls.

\section{Acknowledgements}

We are indebted to Dr. Maria Giżewska for her help in organization of this study.

Supported by the Medical Faculty, University of Rzeszów (Poland).

\section{REFERENCES}

Brown MCJ, Guest JF (1999) Economic impact of feeding a phenylalanine restricted diet to adults with previ- 
ously untreated phenylketonuria. I Intellect Disabil Res 43: 30-37.

Chalmers RA, Lawson AM (1975) Human metabolic diseases. Chem Brit 11: 290-295.

Collin C, Wade DT, Davies S, Horne V (1988) The Barthel ADL Index: a reliability study. Int Disabil Studies 10: 61-63.

Dalek B, Czernicki J (1999) Tests on the evaluation of elderly patient's functional fitness. Physiotherapy 7: 47-51 (in Polish).

Fitzgerald B, Morgan J, Keene N, Rollinson R, Hodgson A, Dalrymple-Smith J (2000) An investigation into diet treatment for adults with previously untreated phenylketonuria and severe intellectual disability. J Intellect Disabil Res 44: 53-59.

Franssen EH, Souren LE, Torossian CL, Reisberg B (1999) Equilibrium and limb coordination in mild cognitive impairment and mild Alzheimer's disease. J Am Geriatr Soc 47: 463-469.

Giżewska M (2006) PKU as a cause of intellectual disability in the adult before the era of newborns screening tests-phenylalanine neurotoxic mechanism. Family Med Primar Care Rev 2: 386-391.

Giżewska M, Ołtarzewski M, Sendecka E (2003) Patients with PKU born before introducing screening tests: various faces of the same disease. Adv Pediatrics 2: 24-32 (in Polish).

Hsueh J-P, Lin J-H, Jeng J-S, Hsieh (2002) Comparison of the psychometric characteristics of the functional independence measure, 5 item Barthel index, and 10 item Barthel index in patients with strokes. J Neurol Neurosurg Psychiatr 73: 188-190.

Ishimaru K, Tamasawa N, Baba M, Matsunaga M, Takeg K (1993) Phenyloketonuria with adult-onset neurological manifestation. Clin Neurol 33: 961-965.

Kasim S, Moo LR, Zschocke, Jinnah HA (2001) Phenylketonuria presenting in adulthood as progressive spastic paraparesis with dementia. J Neurol Neurosurg Psychiatr 71: 795-797.

Lee PJ, Amos A, Robertson L, Fitzgerald B, Hoskin R, Lilburn M, Weetch E, Murphy G (2009) Adults with late diagnosed PKU and severe challenging behavior: $a$ randomized placebo-controlled trial of phenylalanine - restricted diet. J Neurol Neurosurg Psychiatr 80: 631635.

Mahoney FI, Barthel D (1965) Functional evaluation: the Barthel Index. Maryland State Med J 14: 56-61.

Merrick J, Aspler S, Schwarz G (2003): Phenylalanine-restricted diet should be life-long. A case report on longterm follow-up of an adolescent with untreated phenylketonuria. Int J Adol Med Health 2: 165-168.

Robertson L (2006) The National Society for Phenylketonuria (NSPKU) http://www.nspku.org/Documents/Conf_abstracts/Conference_Abstracts_2006.pdf

Rolling J (1984) The discovery of phenylketonuria. Acta Peadiatr (Suppl) 407: 4-10.

Sansom C (1999) Tandem mass spectrometry: the tool of choice for diagnosing inborn errors of metabolism. Mol Med Today 5: 95-96.

Tinetti ME (1986) Performance-oriented assessment of mobility problems in elderly patients. J Am Geriatr Soc 34: 119-126.

Twardowska-Rajewska J (2006) Short programme of making efforts to streamline seniors in order to minimize balance disturbances. Preliminary reports. Polish Gerontology 1: 41-45 (in Polish).

Yannicelli S, Ryan A (1995) Improvements in behavior and physical manifestations in previously untreated adults with phenylketonuria using a phenylalanine-restricted diet: national survey. J Inherit Metab Dis 18: 131-134.

Weglage J, Oberwrittler C, Marguardt T, Schellscheidt J, von Teeffelen-Heithoff A, Koch G, Gerding H (2000) Neurological deterioration in adult phenylketonuria. $J$ Inherit Metab Dis 23: 83-84.

Williams K (1998) Benefits of normalizing plasma phenyloalanine: Impact on behaviour and health. A case report. J Inherit Metab Dis 8: 785-790.

Sansom C (1999) Tandem mass spectrometry: the tool of choice for diagnosing inborn errors of metabolism. Mol Med Today 5: 95-96.

Zekanowski C, Perez B, Desviat LR, Wiszniewski W, Ugarte $\mathrm{M}$ (2000) In vitro expression analysis of R68G and R68S mutations in phenylalanine hydroxylase gene. Acta Biochim Polon 47: 365-369. 